\title{
Ovarian Endometrioid Stromal and Related Neoplasms
}

National Cancer Institute

\section{Source}

National Cancer Institute. Ovarian Endometrioid Stromal and Related Neoplasms. NCI

Thesaurus. Code C40065.

A category of rare neoplasms that arise from the ovary. It includes low grade

endometrioid stromal sarcoma and undifferentiated sarcoma. 